\title{
Compensatory Transfers in Two-Player Decision Problems
}

\section{Citation}

Green, Jerry R. 2005. Compensatory transfers in two-player decision problems. International Journal of Game Theory 33(2): 159-180.

\section{Published Version}

http://dx.doi.org/10.1007/s00182-005-0200-1

\section{Permanent link}

http://nrs.harvard.edu/urn-3:HUL.InstRepos:3204680

\section{Terms of Use}

This article was downloaded from Harvard University's DASH repository, and is made available under the terms and conditions applicable to Other Posted Material, as set forth at http:// nrs.harvard.edu/urn-3:HUL.InstRepos:dash.current.terms-of-use\#LAA

\section{Share Your Story}

The Harvard community has made this article openly available.

Please share how this access benefits you. Submit a story.

\section{Accessibility}



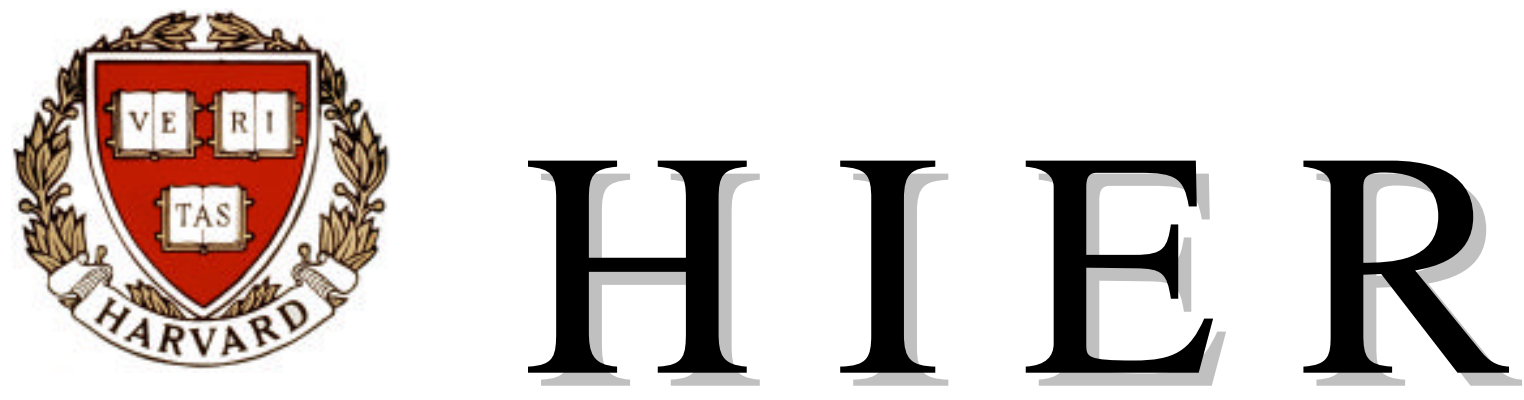

\section{Harvard Institute of Economic Research}

Discussion Paper Number 2015

Compensatory Transfers in Two-Player

Decision Problems

by

Jerry R. Green

August 2003

\section{Harvard University \\ Cambridge, Massachusetts}

This paper can be downloaded without charge from:

http://post.economics.harvard.edu/hier/2003papers/2003list.html

The Social Science Research Network Electronic Paper Collection:

http://ssrn.com/abstract=437161 


\title{
Compensatory Transfers in Two-Player Decision Problems
}

\author{
Jerry R. Green \\ Harvard University
}

first version: August 2, 2002

this version: February 3, 2003

\section{Abstract}

This paper presents an axiomatic characterization of a family of solutions to two-player quasi-linear social choice problems. In these problems the players select a single action from a set available to them. They may also transfer money between themselves.

The solutions form a one-parameter family, where the parameter is a nonnegative number, $t$.

The solutions can be interpreted as follows: Any efficient action can be selected. Based on this action, compute for each player a "best claim for compensation". A claim for compensation is the difference between the value of an alternative action and the selected efficient action, minus a penalty proportional to the extent to which the alternative action is inefficient. The coefficient of proportionality of this penalty is $t$. The best claim for compensation for a player is the maximum of this computed claim over all possible alternative actions. The solution, at the parameter value $t$, is to implement the chosen efficient action and make a monetary transfer equal to the average of these two best claims.

The characterization relies on three main axioms. The paper presents and justifies these axioms and compares them to related conditions used in other bargaining contexts. In Nash Bargaining Theory, the axioms analagous to these three are in conflict with each other. In contrast, in the quasi-linear social choice setting of this paper, all three conditions can be satisfied simultaneously.

Acknowledgement: This work was supported by the Division of Research at the Harvard Business School. Thanks are due to the Cowles Foundation for Research in Economics at Yale University for its kind hospitality during the Spring of 2002. I have received helpful advice and comments from Youngsub Chun, Ehud Kalai, Herve Moulin, Al Roth, Ilya Segal, Adam Szeidl, Richard Zeckhauser, and other members of the Theory Seminars at Harvard, MIT, Princeton, Rice and Northwestern. 


\title{
Compensatory Transfers in Two-Player Decision Problems
}

\author{
Jerry R. Green \\ Harvard University
}

\section{Introduction}

\subsection{The Problem}

This paper concerns the normative analysis of two-player quasi-linear social choice problems. In these problems, two players select a single action from a set available to them. In addition, the players may make a payment of money to one another. Preferences are quasi-linear in this monetary transfer - relative evaluations of the outcomes are independent of the amount of money paid or received.

\subsection{Solutions}

A solution is a function that determines the action to be implemented and the payment to be made in each quasi-linear social choice problem. We seek solutions that always result in efficient outcomes and that have additional normative properties. In quasi-linear social choice problems, efficiency requires that the chosen action maximizes the sum of the players' evaluations; finding such actions is a straightforward calculation. The more subtle question therefore concerns how the monetary payments should vary with the problem that is faced.

We present an axiomatic structure that characterizes a one-parameter family of solutions. The parameter, denoted $t$, can take any non-negative value. The solutions and the interpretation of $t$ are easy to describe:

Select any efficient action ${ }^{1}$ and implement it. Then consider each player in turn. For the first player, and for each possible action, make the following computation: Take the difference between his evaluation of this action and the implemented action. Subtract $t$ times the amount by which this action is inefficient - that is the quantity of money which, if added to the combined payoff of the two players, would make this action as efficient as the one that has been selected. Maximize this difference over all the actions ${ }^{2}$. Think of the resulting quantity as the first player's proposal for a transfer of money in

\footnotetext{
${ }^{1}$ If there is more than one, any can be selected. The solution, in the utility space, will be invariant to this selection.

${ }^{2}$ The maximal difference must be non-negative because if the action in question were equal to the implemented action, both terms would be zero.
} 
his favor. Now make the same calculation for the second player; think of the result as the second player's proposal. The solution, at the parameter value $t$, is to implement the selected efficient action, and to make a monetary transfer equal to the average of these two proposals.

\subsection{Interpretation of Solutions and the Parameter $t$}

The procedure outlined above gives a method for calculating the result of any solution obeying the axioms we propose. It describes this result as if there were a procedure for making and adjudicating claims for compensation. Yet the approach of this paper is entirely normative. The axioms describe desirable qualitative properties of solutions and do not mention "claims", "compensation" or "proposals" in any way. These terms help us understand the solutions and elucidate their behavior as the problem varies but are not part of the theory itself.

Monetary payments can be interpreted as compensation paid by one player to another for the latter's having forgone the opportunity to chose a different decision - one that this latter player would have preferred but which is inefficient for the group as a whole. For this reason we call the monetary payment a "compensatory transfer". In general, as can be seen from the calculation described above, both players may have a justifiable claim for a compensatory transfer.

The parameter $t$ represents a quantitative measure of the influence of "inefficient forgone alternatives" on the recommended result. A high value of $t$ means that the solution tends to be less sensitive to such alternatives, and transfers will tend to be small in absolute value. A low value of $t$ means that a player will be well-compensated when his favorite alternative is not selected. At $t=0$ the transfer will depend only on the maximal evaluation that each of the two players gives to any action. ${ }^{3}$

$>$ From the nature of the calculation method described, another property of solutions can easily be seen. The solution will depend only on the single "best proposal" that each player has available. The addition, deletion, or modification of actions that do not affect these two "best proposals" or the efficient selected action will necessarily leave the recommended outcome unchanged.

\subsection{Applications}

Quasi-linear social choice problems have a wide range of application. Cost allocation is one important area where they have been used ${ }^{4}$. In cost allocation problems, the payoffs are usually thought of as negative - efficient actions are those that result in the least negative aggregate payoff.

\footnotetext{
${ }^{3}$ As discussed below in sections 1.6 .4 and 6.1 , the solution at $t=0$ does not survive a natural strengthening of our continuity axiom. It is the only member of the one-parameter family characterized in Theorem 13 that fails this test. Thus, in some sense, this solution is a limiting case of the solutions with $t>0$ which are to be preferred on theoretical grounds. See Theorem 16.

${ }^{4}$ See Moulin[24] and Young[52], for a full discussion. Also Moulin[21] in Arrow, Sen and Suzumura[1], Champsaur[5], Kaneko[17], Loehmann and Whinston[18], and Chun[7][8][9].
} 
Many collective decisions in multi-division businesses or in multi-jurisdiction governmental settings fit naturally into the framework of this paper. The players are divisions, localities, or administrative units. One level higher in the organization than these "players" is a central authority that would like the players to take efficient decisions and to allocate the costs and benefits of these decisions equitably and consistently across problems. A related application is the allocation of corporate profits to divisions for reporting purposes.

We are also interested in using this model to evaluate the behavior of actual pairs of bargainers and of individuals who are called upon to make ethical judgments concerning problems faced by others. One of the advantages of having described a one-parameter family of solutions is that we can use experimental data to test the model ${ }^{5}$. If the model predicts well, we can then estimate the parameter value that is seemingly being used by pairs of bargainers or individuals whose behavior conforms to the theory. ${ }^{6}$

\subsection{Axiomatic Structure}

In addition to the standard postulates such as symmetry, three other axioms are used to characterize a family of solutions. The first is additivity: Solving independent problems should produce the same outcome whether they are approached separately or jointly. The second is a form of monotonicity: Consider an action that is better for one of the players than any of the efficient actions. The existence of such an action creates an argument for a compensatory transfer in favor of this player. This argument should become stronger the less inefficient is the action.

The third axiom is called Recursive Invariance. ${ }^{7}$ Recursive Invariance contemplates a situation in which the solution is recommending that a transfer be made in order to reach a utility allocation that is not directly feasible by the choice of an action ${ }^{8}$. In such a situation the axiom states that the addition of a new feasible action that happened to produce the solution's recommended utility allocation, without the need for a monetary transfer, should have no effect: the same utility allocation should be implemented. It could be reached either by retaining the original action-transfer pair, or by adopting the new action and making no transfers.

After formally presenting the axioms, we will argue that a failure of this invariance property would render the solution "vulnerable to renegotiation".

Recursive invariance is not directly comparable to any axioms used in other

\footnotetext{
${ }^{5}$ See, for example, Bar-Hillel and Yaari[4].

${ }^{6}$ See Chugh, Green and Idson[6].

${ }^{7}$ This axioms has only been stated once before in the literature. See Chun [8]. Chun's term for this axiom is Trivial Independence. He shows that, in combination with Pareto Efficiency, it is implied by a variety of other axioms. Chun did not offer any separate justification for Trivial Independence. His paper was concerned with monotonicity and other comparative static properties of solutions, not with additivity. Trivial Independence was shown to be a logically weaker assumption than other postulates that were needed for his main theorem and was thus not separately explored.

${ }^{8}$ Or a mixture of feasible actions.
} 
approaches to bargaining theory although it is similar in spirit to "consistency" and "independence" axioms ${ }^{9}$.

\subsection{Relationship to Prior Work}

\subsubsection{Normative Bargaining Theories}

The normative bargaining literature falls into three groups. First, there is the Nash [29] approach, in which a feasible set of utility allocations and a status quo (or disagreement pont) comprise the data of the problem. Second, there is the approach of games in characteristic function form ${ }^{10}$, in which the data of the problem are the total utilities that each subset of the players can achieve on its own. Third, there is the quasi-linear social choice framework. ${ }^{11}$

The Nash framework allows the feasible set of utility allocations to be an arbitrary convex set. This allows for risk-averse players. In contrast, in the quasilinear framework and in games in characteristic function form, risk-neutrality with respect to monetary transfers is assumed.

The disagreement point plays a special role in the Nash framework ${ }^{12}$. In games in characteristic function form, the utility outcome where each player obtains the payoff he would receive if he were acting alone frequently plays the role of the disagreement payoff. In contrast, in the quasi-linear social choice model, if there were a status quo ante representing the situation before bargaining begins, implementing that point and making no transfer of money is treated on a par with all the other possible outcomes. It is not regarded as a "threat point", or given any other special role.

Quasi-linear social choice problems embody a two-level treatment of the utility possibility set. Some of the allocations result directly from the choice of an action, with no transfers being made. Other allocations can be reached only with non-zero transfers. The utility possibility set is a half-space - the set of all utility allocations whose sum is less than or equal to the maximal amount that can be achieved. The solution, lying on the boundary of this half-space, depends on the set of utility allocations that are reachable without the use of transfers. In this sense, the utility allocations that are induced directly by the choice of an action enjoy a different status, and have more salience within the theory, than those that are reached by the superposition of a monetary transfer upon a real decision. This distinction between the way in which feasible utility

\footnotetext{
${ }^{9}$ Classic references on consistency and related ideas include Aumann and Maschler[3], Moulin[27], Peters[30], Roth[34], Schmeidler[38], Sobolev[40], and Thomson[43][47].

${ }^{10}$ We will restrict attention here to the transferable utility case. In this case, there is the implicit assumption of quasi-linearity of utilities - although the underlying set of actions and their evaluations are not usually mentioned in the analysis. See, for example, Aumann[2], and Roth[35]. For a discussion of games in characteristic function form without transferable utilities see Hart[14].

${ }^{11}$ These three approaches operate directly in the utility space. There is a fourth family of models as well - those that use information on how a utility allocation is achieved, as well as on what the utilities are. See Roemer [33] for an extensive discussion.

12 See Peters[30] and Schmitz[37].
} 
allocations are attained is not used in either the Nash-type theories or in games in characteristic function form.

While the two-level treatment of the feasible set might seem contrary to the utilitarian tradition, it is an essential part of the formulation of the problem we have posed. Consider the simplest possible problems in our domain - those in which there is only one possible action. A solution to these problems should not impose any monetary transfer because the role of transfers is to create equitable compromises based on the relationships between the action that is actually chosen and other actions that could have been chosen. However, in these simple problems the utility-possibility set is a half-space, as always. Only in the presence of inefficient foregone actions is there a need for transfers, and hence the theoretical necessity for our two-level treatment of the utilitypossibility set.

The use of distributional information is another way in which the analysis of games in characteristic function form differs from that considered here. Each coalition is summarized by a single number, representing the best that that group can do acting on its own. What is not stated or used in the analysis is what the distribution of this maximal payoff among the members of the coalition would be.

To summarize: Games in characteristic function form use information about payoffs for independent subgroups, whereas our analysis uses only the possibilities for both players acting in concert. Our theory neglects "threat points" or other results obtainable by individuals acting alone. ${ }^{13}$ At the same time, we do use information about the individual player's payoffs that would arise at all the decisions that could be made - both the inefficient and the efficient actions - whereas this distributional information is irrelevant to the analysis of games in characteristic function form.

\subsubsection{Comparison of Axiomatic Structures and Results Obtained}

The axioms in this paper are related to axioms that have been used elsewhere in the normative bargaining literature, either in the Nash or characteristic function frameworks, or in quasi-linear social choice theory. A full discussion will be given below, after the axioms have been presented formally. At this point, we will only highlight the differences in the results obtained.

The major difference between the results in this paper and those of other models, either those in the Nash framework or in games in characteristic function form, is that in the present context all the axioms can be simultaneously satisfied. In the Nash framework, the additivity axiom and the monotonicity axiom are in direct conflict, in the presence of the other standard axioms. An axiom related to additivity is used to characterize the superadditive solution, see Maschler and Perles [19]. Monotonicity is used to characterize the

\footnotetext{
${ }^{13}$ Theories that are intended for application in organizations that have longevity, where many decisions will be faced over time that will be handled the same way, should not be based on threats to defect and destroy the organization. See the remarks below in Section 1.6.3 on local and situational justice.
} 
Kalai-Smorodinsky solution, Kalai and Smorodinsky [16]. These solutions are different; thus the axioms are contradictory ${ }^{14}$.

In games in characteristic function form, the monotonicity axiom cannot be stated precisely in the form we use it. Nevertheless, an informal comparison of the spirit of the results can be given. For games in characteristic function form, Young[51] has shown that a form of monotonicity, called strong monotonicity, can be used to characterize the Shapley Value. Indeed, this characterization does not make use of additivity - which was Shapley's route to this solution. In our framework, monotonicity does not imply additivity. However, as in the case of the Shapley Value, the two properties can be satisfied simultaneously.

In the framework of this paper, monotonicity and additivity characterize a fairly large set of solutions - see Theorem 12. One additional axiom, Recursive Invariance, narrows the set of solutions down further to the one-parameter family discussed above. This is the main result - see Theorem 13. A natural strengthening of the continuity requirement eliminates only one of these solutions - see Theorem 16 .

Theorem 18 presents the interpretation of the solutions characterized in Theorem 13 in terms of averages of the best proposals of the players, adjusting these proposals in proportion to the level of inefficiency that would be entailed. This interpretation gives rises to the tax-based formula described above.

\subsubsection{Relationship to Social Choice Theory}

The type of collective decision problem considered here is very different from that in much of classical social choice theory. The problems for which our theory is appropriate are those in which the decision to be taken is isolated from other factors affecting the players involved. In contrast, social choice theory takes a more global perspective. Particular decisions are not isolated. Everything is compressed into the idea of an all-inclusive "social state". These social states can be evaluated by the players, and this evaluation is interpreted within social choice theory as the players' overall welfare level. In social choice theory, typical axioms relate to how an individual evaluates his or her own welfare. Some theories allow for comparisons of one's own welfare to that of others, or to the welfare that would be achieved if the real outcomes that are given to others were instead received by the player in question. Achieving fairness, efficiency and consistency, across both people and situations, are the goals. By abstracting from the particular details of a social decision problem, social choice theory gains its strength, generality and normative force.

In contrast to social choice theory, the theory developed here does not make reference to overall outcomes. The idea is simply to be fair "locally" - using as a reference only the outcomes relevant to the decision at hand. How well or badly situated the participants are in the remainder of their experiences is not a part of this analysis ${ }^{15}$. The idea is only to be fair in so far as the decision at

\footnotetext{
${ }^{14}$ In the presence of the additional requirements for efficiency and invariance to the numerical representation of the preference ordering.

${ }^{15}$ Indeed, since the "players" may not be comparable to each other comparative equity
} 
hand is concerned. For this reason, Elster [11], Sen [39], Young [50] and others have used the terms "local justice" or "situational justice" to describe the spirit of models such as the one presented in this paper.

\subsubsection{Prior Work on Quasi-linear Social Choice Problems}

Prior work on quasi-linear social choice problems has identified several classes of solutions. With one exception, all these solutions differ from those in this paper. The exception is the solution called Equal Allocation of Non-Separable Alternatives (EANS), which has a long history in the context of cost allocation ${ }^{16}$. This solution corresponds to the special case of $t=0$ in our one parameter family of solutions, as characterized in Theorem 13 and interpreted in Section $5^{17}$.

As it turns out, the EANS solution is the only member of the family we characterize that is eliminated by the strengthening of our continuity axiom in Theorem 16. All the solutions corresponding to $t \in(0, \infty)$ satisfy this stronger continuity axiom - but they will in general differ from EANS.

Both Moulin[23] and Chun[7] formulate axioms that focus on the behavior of the solutions as the number of participants vary. In their models the set of actions is fixed, although the players' evaluations of these actions can vary. Dubins[10] uses an axiom that relates to the incentive properties that solutions would possess if they were played as non-cooperative games. With the sole exception of Equal Allocation of Non-separable Surplus, the solutions determined in these papers do not satisfy the additivity hypothesis discussed above.

In contrast to these approaches, the solutions defined in this paper are explicitly meant to apply only to two-player situations in which the outcome is determined cooperatively, with full information about the payoffs and therefore no incentive problems. The number and nature of the actions is variable across problems and is not constrained.

\subsection{Outline of This Paper}

Section 2 presents the model. Section 3 presents the axioms and discusses them in more detail. Properties of solutions are presented in Section 4, which contains all the principal results. In Section 5 we interpret these results in terms of "claims", adjusted for "inefficiency" by means of "taxes", as discussed above. A geometrical construction of solutions is also given in this section. Section 6 collects various comments and comparisons to other work and discusses extensions and open problems arising from this research. Proofs of two main results are in Section 7. Proofs of the other lemmas and theorems are either straightforward or follow immediately from the discussion in the text. Several figures follow.

considerations may not be relevant, or even meaningful. For example, the players may be divisions of a firm with entirely distinct functional responsibilities, very different in size or in their composition of employment.

${ }^{16}$ See Ransmeier[32], Straffin and Heaney[41], and Moulin[27].

${ }^{17}$ See the remarks about the solution with $t=0$ in Section 1.3 and footnote 3 above. 


\section{The Model}

The two players are denoted by $i=1,2$. A problem is a set $B \subset \mathbb{R}^{2}$ that is closed, convex, comprehensive and bounded above. The set of all problems is denoted $\mathcal{B}$.

For each $B \in \mathcal{B}$, let $\bar{x}(B)=\max _{x \in B} x_{1}+x_{2}$. Let $H(B)=\left\{z \in \mathbb{R}^{2} \mid z_{1}+z_{2}=\right.$ $\bar{x}(B)\}$.

A solution is a function $f: \mathcal{B} \rightarrow \mathbb{R}^{2}$.

The interpretation of a solution $f$ is that when $f$ is applied to a problem $B$, the final utilities received by the players are $f(B)=\left(f_{1}(B), f_{2}(B)\right)$. Our first axiom, efficiency, will require that $f(B) \in H(B)$. The utility outcome $f(B)$ can be achieved by selecting an action producing $x \in B \cap H(B)$ and by making a transfer of money $t=\left(t_{1}, t_{2}\right)$, with $t_{1}=-t_{2}$, such that $f_{i}(B)=x_{i}+t_{i}$, for $i=1,2$. 


\section{Axioms}

If $\xi=\left(\xi_{1}, \xi_{2}\right) \in \mathbb{R}^{2}$ denote by $\pi(\xi)$ the vector $\left(\xi_{2}, \xi_{1}\right) \in \mathbb{R}^{2}$. Similarly, if $X \subset \mathbb{R}^{2}$, denote by $\pi X$ the set $\left\{\left(\xi_{1}, \xi_{2}\right) \in \mathbb{R}^{2} \mid \pi \xi \in X\right\}$.

If $X \subset \mathbb{R}^{2}$ and $X$ is bounded above, define the comprehensive hull of $X$ as the smallest set in $\mathcal{B}$ that contains $X$, and denote it by $K(X)$.

The first four axioms are entirely standard:

Axiom 1 Efficiency (E): A solution $f$ satisfies efficiency if for all $B \in \mathcal{B}$, $f(B) \in H(B)$.

Axiom 2 Anonymity $(A N)$ : A solution $f$ satisfies anonymity if for all $B \in \mathcal{B}$, $\pi f(B)=f(\pi B)$.

Axiom 3 Continuity $(C)$ : A solution $f$ satisfies continuity if it is continuous in the Hausdorff topology on $\mathcal{B}$.

Axiom 4 Independence of Utility Origins (IU): A solution $f$ satisfies independence of utility origins if for all $x \in \mathbb{R}^{2}$, and all $B \in \mathcal{B}, f(B+\{x\})=f(B)+x$.

Efficiency, anonymity and continuity require no further comment. Independence of utility origins expresses the idea that the solution should depend on the relative evaluation that each individual has of the various actions. The evaluation scale used in a numerical representation of a quasi-linear utility function is determined only up to an additive constant. This constant should not affect the real aspects of the solution - the selection of the action to be taken and the transfers to be made ${ }^{18}$.

The first question one might ask is whether compensatory transfers should be paid at all. Why not simply select (the midpoint) of the set of efficient alternatives $B \cap H(B)$ and leave it at that?

This "no transfer solution" is eliminated by the four axioms above. Consider a sequence of problems with only two actions, an efficient action which remains fixed, and an inefficient action which is improved throughout the sequence until, in the limit, its payoff converges to a different point than the efficient action, but one that is equally efficient. If throughout this sequence no transfers were paid the solution would be at the efficient point. By continuity, in the limiting case it would remain at this point. However, by anonymity and independence of utility origins, the solution in the limiting case should be the average of the payoffs of the two actions. Never making any transfers would thus result in a discontinuity at this limit. Positive monetary transfers must be paid in at least some cases.

\footnotetext{
${ }^{18}$ Independence of Utility Origins plays the same role in our theory as Independence of Linear Transformations of Utility Scales plays in Nash-type theories. Numerical representation of the preferences, incorporating free parameters with no behavioral meaning, should not affect the real bargaining results.
} 
We will present three more axioms, which together with the four axioms above will characterize a one-parameter family of solutions. These axioms, Additivity, Monotonicity, and Recursive Invariance, will be imposed seriatim, in this order. At each step, we obtain a family of solutions that are easy to describe. The first axiom is well known; the second is closely related to monotonicity axioms used in other bargaining models but has not been previously applied in quasi-linear social choice theories; and the third has been mentioned in the literature only once before, but has not been used in a characterization theorem ${ }^{19}$.

Axiom 5 Additivity $(A D)$ : A solution $f$ satisfies additivity if for all $B_{1}, B_{2} \in$ $\mathcal{B}, f\left(B_{1}+B_{2}\right)=f\left(B_{1}\right)+f\left(B_{2}\right)$.

The additivity axiom is commonly used in games in characteristic function form, where it characterizes the Shapley Value. A related axiom, called superadditivity, is used in Nash Bargaining Theory, where for the two person case it characterizes the Maschler-Perles [19] solution. ${ }^{20}$

The justification for additivity is based on considering those special pairs of problems that do not interact at all. In such pairs, preference over actions in each problem are invariant to the action taken in the other. Pairs of noninteracting problems can be combined into a composite problem. Each action in the composite problem is actually a pair of actions, one chosen in each of the original problems. Additivity is simply the requirement that the players should be indifferent as to whether independent problems are solved separately or jointly. Further justification for this axiom is presented in Myerson[28], Roth[34], Maschler and Perles[19] and Peters[31].

Axiom 6 Monotonicity $(M)$ : A solution $f$ satisfies Monotonicity if $f_{1}(K(\{(0,0)\} \cup$ $\{(1,-x)\})$ ) is non-increasing in $x$, for $x \geqq 1$.

Monotonicity is related in spirit to various monotonicity axioms in the bargaining literature ${ }^{21}$, but has different implications arising from the structure of quasi-linear social choice problems. The intent of this axiom is capture the ethical spirit of a process in which monetary transfers are paid to a player who can make a good argument, to a neutral observer, that he has "forgone" the prospect of decisions that would have been better for him, in favor of one of the collectively efficient decisions. Such an argument should be stronger when the forgone alternative is less inefficient, but no less beneficial to this player.

\footnotetext{
${ }^{19}$ See footnote 7 above.

${ }^{20}$ However, this solution exists only for the case of $n=2$, see Maschler and Perles[20]. On the other hand, in the present model, the axioms E, AN, C, IU, and AD can be satisfied for any $\mathrm{n}$. Indeed there are infinitely many solutions that do so, see Green[12].

${ }^{21}$ See Kalai-Smorodinsky [16], Moulin[27], Roth[36], Thomson[44], Thomson and Myerson[45], and Young[49], [50].
} 
Insert Figure 1 here

Now we come to the final axiom:

Axiom 7 Recursive Invariance (RI): A solution satisfies recursive invariance if for all $B \in \mathcal{B}, f(K(B \cup\{f(B)\}))=f(B)$.

Recursive Invariance is based on the idea that when players agree to use a solution $f$ they are committing themselves to a process that imposes monetary transfers in order to compensate each other for forgoing alternatives that would have been preferred but which might not be efficient. The players are agreeing to make a transfer that results in $f(B)$ recognizing that this allocation may not be feasible through the choice of an action alone. Recursive Invariance embodies the idea that the players already regard $f(B)$ as a fair outcome when the underlying possibilities are $B$. Having committed themselves to a process that results in this outcome, the players should still consider $f(B)$ to be a just result if a means of achieving it directly, without the use of transfers, were added to the feasible outcomes already in $B$.

Recursive Invariance can also be interpreted as a form of renegotiation proofness condition. Imagine that after $B$ becomes known, but prior to the actual implementation of any outcome or transfers, the players enter into a renegotiation. At this stage, given that the players have agreed to use the solution $f$, $f(B)$ has the same status as all the points in the original $B$-it is an outcome that can be selected without the need for any further monetary transfers. If $f(K(B \cup\{f(B)\})) \neq f(B)$ the players would be rejecting the recommendation of the solution $f$ at this stage, renegotiating their original commitment to use $f$ even though nothing real has changed.

Insert Figure 2 here 


\section{Constructing Solutions}

The construction of solutions is based on a simple geometrical idea. Problems in $\mathcal{B}$ can all be written as the sum of sets in a very simple subfamily - the subfamily of $\mathcal{B}$ in which there are only two outcomes. Once the solution is fixed on this subfamily, it can be extended to all of $\mathcal{B}$ using the additivity axiom.

The Monotonicity and Recursive Invariance axioms restrict the behavior of solutions on this subfamily. These restrictions have implications for the behavior of solutions on all of $\mathcal{B}$ which are expressed in the main characterization theorems.

To implement this approach to the construction of solutions, some additional notation will be useful.

Let $\mathcal{B}_{0} \subset \mathcal{B}$ be the set of $B \in \mathcal{B}$ with $\bar{x}(B)=0$, and $(0,0) \in B$.

For $x \geqq \lambda>0$, let $C_{1}(\lambda, x)=K(\{(0,0)\} \cup\{(\lambda,-x)\})$ and $C_{2}(\lambda, x)=$ $K(\{(0,0)\} \cup(-x, \lambda)\})$. The subfamily consisting of all sets $C_{i}(\lambda, x)$ for $i=1,2$ - problems with only two outcomes - will form the basis of solutions on $\mathcal{B}_{0}$. These solutions can then be extended to all of $\mathcal{B}$.

The next three lemmas give precise statements of these ideas.

Any $B \in \mathcal{B}_{0}$ can be approximated by a finite sum of the form $\sum_{j=1, \ldots, J} C_{1}\left(\lambda_{j}^{1}, x_{j}^{1}\right)+$ $\sum_{j=1, \ldots, J} C_{2}\left(\lambda_{j}^{2}, x_{j}^{2}\right)$. An exact decomposition of $B \in \mathcal{B}_{0}$ as a sum of sets each of which is generated by a problem with only two actions is given by:

Lemma 8 For each $B \in \mathcal{B}_{0}$ there exists a pair of non-negative measures $\mu_{1}^{B}, \mu_{2}^{B}$ on $[1, \infty)$ such that

$$
B=\int C_{1}(1, x) d \mu_{1}^{B}(x)+\int C_{2}(1, x) d \mu_{2}^{B}(x)
$$

Anonymity and additivity give rise to the translation invariance of solutions, which can be expressed as:

Lemma 9 If $f$ satisfies $E, A N, C$, and $I U, f(K(\{x\}))=x$ for all $x \in \mathbb{R}^{2}$

As a consequence of Lemmas 8 and 9, given the value of a solution on the sets $C_{i}(1, x)$, for $i=1,2$ and $x \geqq 1$, we can find the solution to any problem $B \in \mathcal{B}$ by translating $B$ by a vector $-x$ such that $x \in B \cap H(B)$, resulting in a set $B_{0} \in \mathcal{B}_{0}$. Then, the solution $f\left(B_{0}\right)$ is computed using Lemma 8 , and the original problem $B$ is therefore solved at $f\left(B_{0}\right)+x$ This process is summarized in Lemma 10:

Lemma 10 If $f: \mathcal{B}_{0} \rightarrow \mathbb{R}^{2}$ satisfies $E, A N, C, I U$ and $A D$ on $\mathcal{B}_{0}$, then $f$ can be uniquely extended to $\mathcal{B}$. 
Given a solution $f$ let $g^{f}:[1, \infty) \rightarrow \mathbb{R}$ be defined by

$$
g^{f}(x)=f_{1}\left(C_{1}(1, x)\right)
$$

Given a function $g:[1, \infty) \rightarrow \mathbb{R}$, Lemma 8 implies that $g$ generates a solution $f$ via the relation:

$$
f_{1}(B)=\int g(x) d \mu_{1}^{B}(x)-\int g(x) d \mu_{2}^{B}(x) \quad \text { for all } B \in \mathcal{B}_{0}
$$

We can characterize a family of solutions $\mathcal{F}$ that are consistent with AN, C, $\mathrm{AD}$ and any additional axioms, by determining the properties that the associated $g^{f}$ must satisfy for any $f \in \mathcal{F}$ under these axioms.

Lemma 11 Given any continuous function $g:[1, \infty) \rightarrow \mathbb{R}$, such that $g(1)=\frac{1}{2}$, there exists a unique solution $f$ satisfying $E, A N, C, I U$ and $A D$ such that $g^{f}(x)=g(x)$ for all $x \in[1, \infty)$. Conversely, if $f$ is a solution satisfying $E$, $A N, C, I U$ and $A D$ then $g^{f}$ is continuous and $g^{f}(1)=\frac{1}{2}$.

Lemma 12 If $f$ is a solution satisfying $E, A N, C, I U, A D$ and $M$, then $g^{f}$ is continuous, non-increasing and $g^{f}(1)=\frac{1}{2}$. Conversely, if $g:[1, \infty) \rightarrow \mathbb{R}$ is any continuous, non-increasing function with $g^{f}(1)=\frac{1}{2}$, the solution $f: \mathcal{B} \rightarrow \mathbb{R}^{2}$ that is generated from $g$, will satisfy $E, A N, A D, C, I U$ and $M$.

Lemma 12 follows directly from the statement of the monotonicity axiom.

The main result of this paper is the characterization of the set of solutions satisfying Recursive Invariance, in addition to the axioms previously imposed:

Theorem 13 If $f$ is a solution satisfying $E, A N, C, I U, A D, M$ and $R I, g^{f}$ : $[1, \infty) \rightarrow \mathbb{R}$ satisfies either:

(i) There exists $x^{*}>1$ such that

$g^{f}(x)=\frac{1}{2}-\frac{1}{2}\left(\frac{x-1}{x^{*}-1}\right) \quad$ for $x \leqq x^{*}$

$g^{f}(x)=0 \quad$ for $x>x^{*}$

or

(ii)

$g^{f}(x)=\frac{1}{2}$ for all $x \in[1, \infty)$. Conversely, all the solutions generated from functions $g$ satisfying (i) and (ii) are consistent with the axioms, E, AN,C,IU,M and $R I$.

The proof of Theorem 13 is in Section 7 . 
The continuity axiom $(C)$ as stated is based on the idea that if payoffs from a given set of actions are perturbed slightly, there should be only a slight change in the resulting utilities. We will now introduce a slight strengthening of the continuity idea with a different motivation: Actions that result in extremely inefficient payoffs should have little weight in determining the outcome. The mathematical way of expressing this idea is as follows:

Take a sequence of sets $B^{i}$. If there exists $B \in \mathcal{B}$ such that for every compact set $X \subset \mathbb{R}^{2}, B^{i} \cap X$ converges in the Hausdorff topology to $B \cap X$, we will say that $B^{i}$ converges to $B$. A topology, which we call the bounded convergence topology, is defined by this family of converging sequences.

The continuity axiom can be strengthened to:

Axiom 14 Axiom $C^{*}$ : The solution $f$ is continuous in the bounded convergence topology.

Lemma 15 If the solution $f$ satisfies $E, A N, C^{*}, I U, A D$ and $M$, then the function $g^{f}$ that represents $f$ satisfies: $\lim _{x \rightarrow \infty} g^{f}(x)=0$

Theorem 16 If $f$ is a solution satisfying $E, A N, C^{*}, I U, A D, M$ and $R I$, then there exists $x^{*} \in(1, \infty)$ such that $g^{f}:[1, \infty) \rightarrow \mathbb{R}$ satisfies:

$$
\begin{array}{ll}
g^{f}(x)=\frac{1}{2}-\frac{1}{2}\left(\frac{x-1}{x^{*}-1}\right) & \text { for } x \leqq x^{*} \\
g^{f}(x)=0 & \text { for } x>x^{*}
\end{array}
$$




\section{Characterizations of Solutions in Terms of Taxes on the Inefficiency of Claims}

In this section we offer an interpretation of Theorem 13 in terms of "best claims for compensation", as discussed in the introduction.

For each $x^{*}>0$ and $x \geqq 0$, let $g^{x^{*}}(x)=\max \left(\frac{1}{2}-\frac{1}{2}\left(\frac{x-1}{x^{*}-1}\right), 0\right)$. Define the solution $f\left(B ; x^{*}\right)$ as the solution generated from $g^{x^{*}}$ by $f_{1}\left(B, x^{*}\right)=$ $\int g^{x^{*}}(x) d \mu_{1}^{B}(x)-\int g^{x^{*}}(x) d \mu_{2}^{B}(x)$.

Take any $x^{0} \in B \cap H(B)$. For each $t \geqq 0$ define

$$
f^{t, x_{0}}(B)=x^{0}+(1 / 2)\left[\left(y_{1}^{*}(B, t),-y_{1}^{*}(B, t)\right)+\left(-y_{2}^{*}(B, t), y_{2}^{*}(B, t)\right)\right]
$$

where $y_{i}^{*}(B, t)$ are the value of the problems

$$
\max _{x \in B}\left(x_{i}-x_{i}^{0}\right)-t\left(\bar{x}(B)-\left(x_{1}+x_{2}\right)\right)
$$

for $i=1,2$.

Lemma 17 The solution $f^{t, x_{0}}(B)$ is independent of the choice of $x^{0} \in B \cap$ $H(B)$.

Therefore we can define $f^{t}(B)=f^{t, x_{0}}(B)$.

Theorem 18 For each $x^{*}>0$, the solution $f\left(B ; x^{*}\right)$ is equal to the solution $f^{t}(B)$ for $t=\frac{1}{x^{*}-1}$.

The solution generated from $g \equiv \frac{1}{2}$ is equal to the solution $f^{0}(B)$.

The limiting case, where $t$ is 0 , corresponds to $x^{*} \rightarrow \infty$ and part (ii) of the conclusion of Theorem 13: $g^{f}(x) \equiv 1 / 2$. In this solution, no decrease in the claim of either player is applied due to the inefficiency of a superior forgone alternative. This is the Equal Allocation of Non-Separable Surplus solution. (See the further discussion in Section 6.1 below.)

The geometry of the solutions are shown in Figure 3. The efficient point is $x^{0}=(0,0)$. Both of the players have superior forgone alternatives. If their payoffs in these two alternatives are adjusted in accordance with the "tax" $t$, their "best claims" for compensation are the points $y^{1}$ and $y^{2}$. The solution, therefore is $f(B)=x^{0}+\frac{1}{2}\left(y^{1}+y^{2}\right)$, as indicated.

Insert Figure 3 here 


\section{Other Remarks}

\subsection{Comparison to Other Solutions}

Moulin[23] discusses three classes of solutions to quasi-linear social choice problems. The first is simply to choose an efficient action and to make no transfers at all. This method necessarily produces discontinuities in the utility outcomes in the neighborhood of problems where there is more than one efficient decision. (See the discussion above in section 3.)

The second class of solutions is called Equal Sharing Above a Convex Reference Level ${ }^{22}$. These methods produce outcomes that depend on the description of the problem in ways other than the set of utilities reached by an action choice. For example, adding other actions whose induced utilities duplicate those already in the feasible set will affect the solution.

The third solution is called, in the cost allocation literature, Equal Allocation of Non-Separable Costs. The EANS solution is obtained by computing best results that can be attained by coalitions of $n-1$ players if they could choose the action without reference to the results of the omitted player: Define $v_{-i}=$ $\max _{x \in B} \sum_{j \neq i} x_{j}$. Then the separable cost (or benefit) ascribed to player $i$ is $s_{i}=\max _{x \in B} \sum_{j=1, \ldots, n} x_{j}-v_{-i}$. The EANS solution gives each player the payoff $s_{i}$ plus an equal share of the difference between the aggregate of these payoffs and the amount available to the group - the "non-separable costs". Thus player $i$ receives $\frac{1}{n}\left[\max _{x \in B} \sum_{j=1, \ldots, n} x_{j}+\sum_{j \neq i} v_{-j}\right]-\frac{n-1}{n} v_{-i}$ under the EANS solution. In the two player situation studied in this paper, the EANS solution gives player $1 \frac{1}{2}\left[\max _{x \in B}\left(x_{1}+x_{2}\right)+\max _{x \in B} x_{1}-\max _{x \in B} x_{2}\right]$. This solution is precisely the solution we obtain when $t=0$.

Take the set of problems defined by $B_{x}=K\{\{(0,0)\},\{(1,-x)\}\}$ for $x>1$. For all these problems, EANS recommends the allocation $(1 / 2,-1 / 2)$, no matter how large $x$ becomes. All the solutions we characterize, for any $t>0$, converge to $(0,0)$ as $x \rightarrow \infty$. We view this convergence as reasonable because a very inefficient alternative such as $(1,-x)$ for $x$ large, should not cause much of a transfer to be paid. This is the justification for Axiom $\mathrm{C}^{*}$ and the resulting restriction to $t>0$ obtained in Theorem 16. (See Figure 4.)

Insert Figure 4 here

\subsection{Testing and Estimating Bargaining Solutions}

This paper has been entirely normative in character. Nevertheless, as is the case with other normative ideas in economics, it is interesting to see if experimenal subjects, faced with this type of problem, behave in a manner consistent with this theory.

In Chugh, Green and Idson[6] we examine whether the presence of inefficient forgone alternatives affects the direction and magnitude of monetary transfers

\footnotetext{
${ }^{22}$ This is class is also explored in some detail by Chun[7][9]
} 
in one-time encounters between pairs of people. We obtain clear evidence for this effect. We are performing also trials where individuals are asked for their normative evaluation about outcomes that are "proposed" for hypothetical problems faced by two other bargainers. We test whether such a judge is wellmodeled by a solution of the form described in this paper, and if so we estimate the value of $t$ that they seem to be using. In future work we hope to show the value of $t$ depends on the context of the bargaining setting and on other social, demographic and behavioral information about the people involved.

\subsection{Cost Allocation Problems}

The model presented above can be extended to cost allocation problems. In cost allocation problems the model is augmented to associate a total cost to every action, in addition to the evaluation that the two players have for the action. Transfers are then required to sum to (the negative) of the cost of the efficient action that is implemented. Thus the financing of the collective decision is incorporated into the system of compensatory transfers that is associated with the selected efficient action.

A problem is a set of points $x=\left(x_{0}, x_{1}, x_{2}\right) \in \mathbb{R}^{3}$, with the interpretation that $x_{0}$ is the negative of the cost of the associated action, and that $x_{i}$ is the benefit of this action to each of the two players, $i=1,2$. The comprehensive hull $B$ of these points in $\mathbb{R}^{3}$ is used to summarize the problem. Under the usual "free disposal" and "probability mixture" hypotheses, allowing costs to be higher and benefits to be lower that those specified at each action, the set of all problems is the family $\mathcal{B}$ of closed, convex, comprehensive subsets of $\mathbb{R}^{3}$ that are bounded above.

Efficiency is the requirement that $x$ maximizes $x_{0}+x_{1}+x_{2}$ over $B$. Let $\bar{x}(B)$ be the value of this maximand, which is the value of the benefits in excess of cost at an efficient action.

A solution is a mapping $f: \mathcal{B} \rightarrow \mathbb{R}^{2}$, such that $f_{1}(B)+f_{2}(B)=\bar{x}(B)$ for all $B \in \mathcal{B}$. This restriction incorporates the sharing of costs into the transfers, as $f_{i}(B)=x_{i}+t_{i}$ and $f_{1}(B)+f_{2}(B)=\bar{x}(B)=x_{0}+x_{1}+x_{2}$ imply $t_{1}+t_{2}=x_{0}$.

The one parameter family of solutions studied above satisfy the natural generalization of all the axioms in this non-zero cost framework. Any efficient action can be selected, resulting in the benefits $x_{i}^{*}$ and the cost $-x_{0}^{*}$. Begin from the payoffs where the cost at the efficient action are shared equally, resulting in payoffs $x_{i}^{*}+\frac{x_{0}^{*}}{2}$. Then, compute the claim $y_{i}^{t}$ of each player according to the value of the problem

$$
\max _{x \in B}\left(x_{i}+\frac{x_{0}}{2}\right)-\left(x_{i}^{*}+\frac{x_{0}^{*}}{2}\right)-t\left(\bar{x}(B)-x_{0}+x_{1}+x_{2}\right)
$$

The solution, at the parameter value $t$ is $\left(f_{1}^{t}(B), f_{2}^{t}(B)\right)=\left(x_{1}^{*}+\frac{x_{0}^{*}}{2}+\right.$ $\left.\frac{y_{1}^{t}-y_{2}^{t}}{2}, x_{2}^{*}+\frac{x_{0}^{*}}{2}+\frac{y_{2}^{t}-y_{1}^{t}}{2}\right)$. 


\subsection{More Than Two Players}

Unlike the extension of our basic model to problems with non-zero costs, the extension to more than two players is anything but trivial. The problem is not that there are no solutions, but rather that there may be others that satisfy the axioms as well.

Consider the subfamily of problems in which all actions are equally efficient and where the maximal possible for the group as a whole is zero. In the case of $n=2$, these problems are trivial: The solution is the midpoint of the efficient segment of $B$.

If we were to apply the idea of the solutions obtained above to the case of $n>2$, the value of $t$ would be irrelevant because all alternatives are equally efficient, and all solutions would give player $i$ the payoff $\frac{1}{n} \sum_{j \neq i} \max _{B} \sum_{k \neq j} x_{k}-$ $\frac{n-1}{n} \max _{B} \sum_{k \neq i} x_{k}$ at every problem in this class ${ }^{23}$.

The solutions that reach this payoff are not, however, the only solutions that satisfy the additivity axiom on this family of problems. One example of a solution that can take a different value on some problems is obtained by defining a game $v$ in characteristic function form as $v(S)=\max _{x \in B} \sum_{i \in S} x_{i}$ and letting the solution be the Shapley value of $v$. Indeed, the results in Green[12] show that there are an infinity of values that can be taken on by additive solutions in this class of problems.

The reason for this difference in the results can be traced to the method of proof used above in the case of $n=2$. We decomposed problems in $\mathcal{B}$ into a sum of problems involving only two actions (recall Lemma 8). Therefore to find solutions satisfying Additivity we needed only to specify the solutions on the very simple subfamily of "two-action" problems, which is the role of the function $g$ above. Once we generated all additive solutions from this function of one variable, the other axioms were used to restrict $g$. It is this idea that does not generalize to larger $n$. In spaces of larger dimension a typical member of $\mathcal{B}$ cannot be written as the sum of such a very simple family of problems. There is, therefore, quite a bit more flexibility available to the "design" of an additive solution. ${ }^{24}$

The Monotonicity and Recursive Invariance axioms deal with problems in which not all actions are efficient. At present it is not known whether all members of the class of solutions identified by Green[12] for the "all-actionsefficient" case can be extended to the general case in a way that preserves Monotonicity and Recursive Invariance. This remains one of the principal questions for further research in this area.

\footnotetext{
${ }^{23}$ Using the constant sum property of the feasible set, $i$ 's payoff can also be written as $\min _{B} x_{i}-\frac{1}{n} \sum_{j=1, \ldots, n} \min _{B} x_{j}$.

${ }^{24}$ Mathematically, the question is related to problems in the theory of convex polytopes. See Grünbaum[13]
} 


\section{Proofs}

Proof of Theorem 13

If $g(y) \equiv \frac{1}{2}$ we have (ii) in the statement of the theorem. If there exists $y$ such that $g(y)>\frac{1}{2}$, we have a violation of the Monotonicity axiom. Assume therefore that there exists $y>1$ such that $0<g(y)<\frac{1}{2}$. By the definition of $g$ we have,

$$
f(K(\{(0,0)\} \cup\{(1,-y)\}))=(g(y),-g(y))
$$

By RI,

$$
f(K(\{(0,0)\} \cup\{(g(y),-g(y))\} \cup\{(1,-y)\}))=(g(y),-g(y))
$$

Express the argument of $f$ on the left hand side of (2) as the sum of two sets in $\mathcal{B}_{0}$ :

$$
\begin{aligned}
& K(\{(0,0)\} \cup\{(g(y),-g(y))\} \cup\{(1,-y)\}) \\
= & K(\{(0,0)\} \cup\{(g(y),-g(y))\})+K(\{(0,0)\} \cup\{(1-g(y), g(y)-y)\})
\end{aligned}
$$

By IU and AN

$$
f\left(K(\{(0,0)\} \cup\{(g(y),-g(y))\})=\left(\frac{g(y)}{2},-\frac{g(y)}{2}\right)\right.
$$

Substituting (3) into (2) and using (4) we have .

$$
f(K(\{(0,0)\} \cup\{(1-g(y), g(y)-y)\}))=\left(\frac{g(y)}{2},-\frac{g(y)}{2}\right)
$$

Now apply the same argument to

$$
K\left(\{(0,0)\} \cup\left\{\left(\frac{g(y)}{2},-\frac{g(y)}{2}\right)\right\} \cup\{(1-g(y), y-g(y))\}\right)
$$

Decompose the argument of (6) as in (3):

$$
\begin{aligned}
& K\left(\{(0,0)\} \cup\left\{\left(\frac{g(y)}{2},-\frac{g(y)}{2}\right)\right\} \cup\{(1-g(y), g(y)-y)\}\right) \\
= & K\left(\{(0,0)\} \cup\left\{\left(\frac{g(y)}{2},-\frac{g(y)}{2}\right)\right\}\right) \\
& +K\left(\{(0,0)\} \cup\left\{\left(1-g(y)-\frac{g(y)}{2}, y-g(y)-\frac{g(y)}{2}\right)\right\}\right)
\end{aligned}
$$

Therefore,

$$
f\left(K\left(\{(0,0)\} \cup\left\{\left(1-g(y)-\frac{g(y)}{2}, y-g(y) \frac{-g(y)}{2}\right)\right\}\right)\right)=\frac{g(y)}{4}
$$


Recursively,

$$
f\left(K\left(\{(0,0)\} \cup\left\{\left(1-g(y)\left(2-\frac{1}{2^{n}}\right), y-g(y)\left(2-\frac{1}{2^{n}}\right)\right)\right\}\right)\right)=\frac{g(y)}{2^{n+1}}
$$

Taking the limit as $n \rightarrow \infty$, we have,

$$
f(K(\{(0,0)\} \cup\{(1-2 g(y), y-2 g(y))\}))=0
$$

or

$$
(1-2 g(y)) g\left(\frac{y-2 g(y)}{1-2 g(y)}\right)=0
$$

Now, since $0<g(y)<\frac{1}{2}$,

$$
g\left(\frac{y-2 g(y)}{1-2 g(y)}\right)=0
$$

Let

$$
x^{*}=\frac{y-2 g(y)}{1-2 g(y)}
$$

Taking $n$ large and recalling that $1<y$ and $0<g(y)<\frac{1}{2}(9)$ implies that $g(x)>0$ for a sequence of points $x^{n}$ approaching $x^{*}$ from below. Therefore, by virtue of the Monontonicity axiom,

$$
x^{*}=\inf \{x \mid g(x)=0\}
$$

Beginning this argument from any $y$ such that $g(y)>0$, we see that (13) holds independent of the value of $y$ selected. Thus,

$$
x^{*}=\frac{x-2 g(x)}{1-2 g(x)} \text { for all } x<x^{*}
$$

Solving (15) over its domain of validity, we obtain,

$$
g(x)=\frac{1}{2}-\frac{x-1}{2\left(x^{*}-1\right)} \text { for all } x<x^{*}
$$

Thus, for the case where there exists $y$ with $g(y)<\frac{1}{2}$, it remains only to show that $g(x)=0$ for $x>x^{*}$. Suppose to the contrary that there exist $y>x^{*}$ with $g(y)<0$. Now (2)] holds independent of the sign of $g(y)$. However, because $g(y)<0$,

$$
\begin{aligned}
& K(\{(0,0)\} \cup\{(g(y),-g(y))\} \cup\{(1,-y)\}) \\
= & K(\{(0,0)\} \cup\{(g(y),-g(y))\})+K(\{(0,0)\} \cup\{(1,-y)\})
\end{aligned}
$$

Applying $\mathrm{AD}$ and $\mathrm{AN}$,

$f(K(\{(0,0)\} \cup\{(g(y),-g(y))\} \cup\{(1,-y)\}))=\left(\frac{g(y)}{2},-\frac{g(y)}{2}\right)+(g(y),-g(y))$

which contradicts (2). 


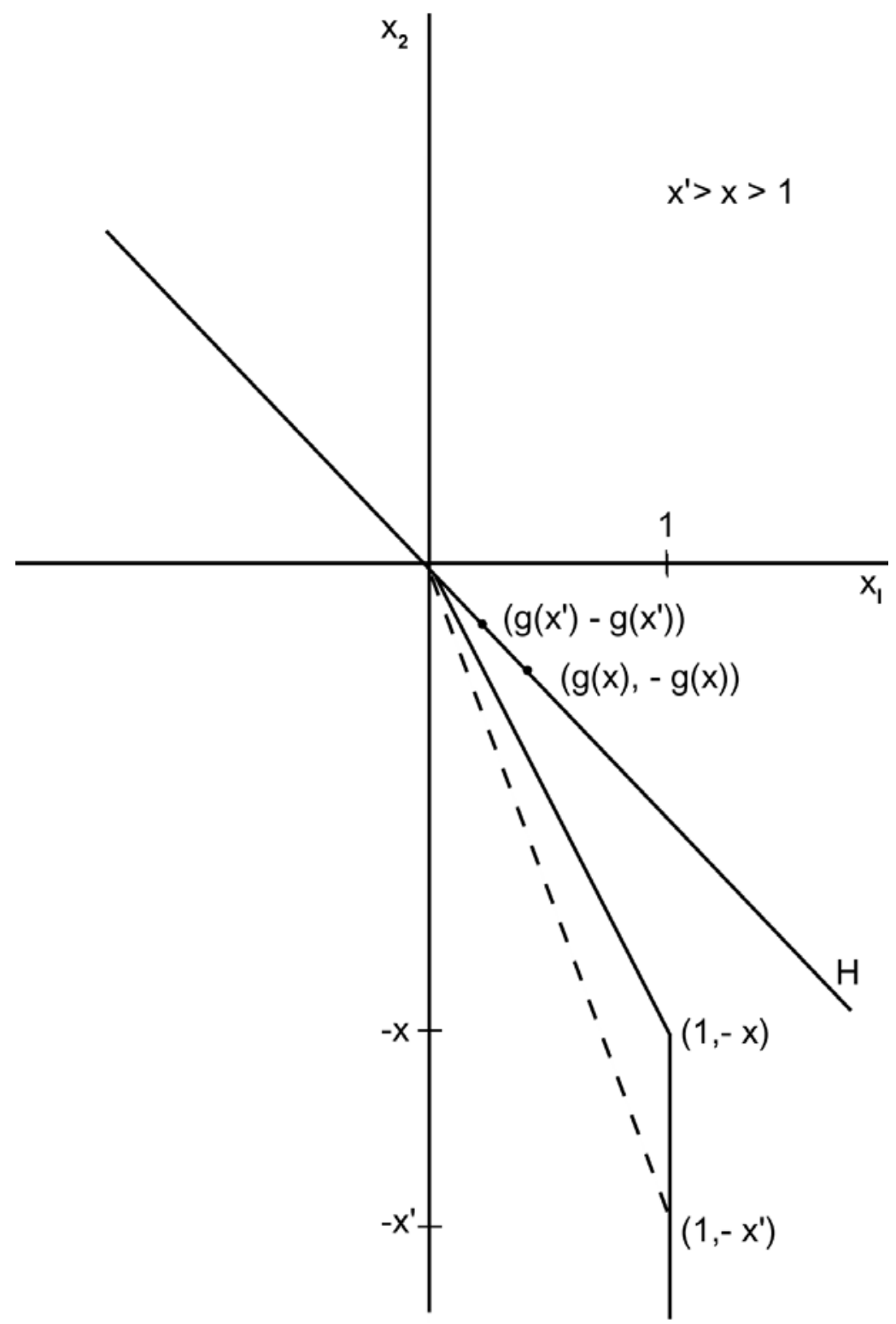

Figure 1 


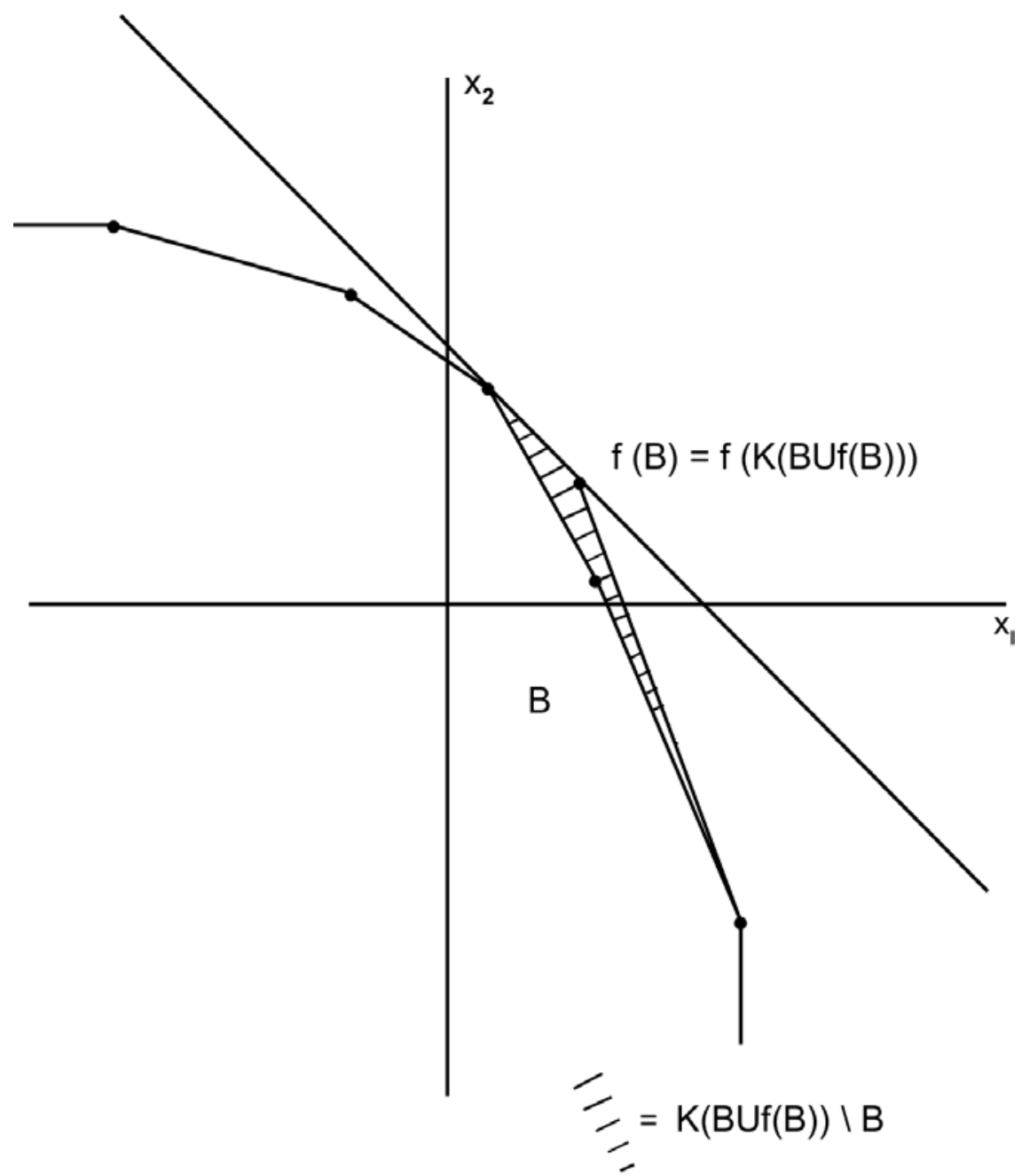

Figure 2 


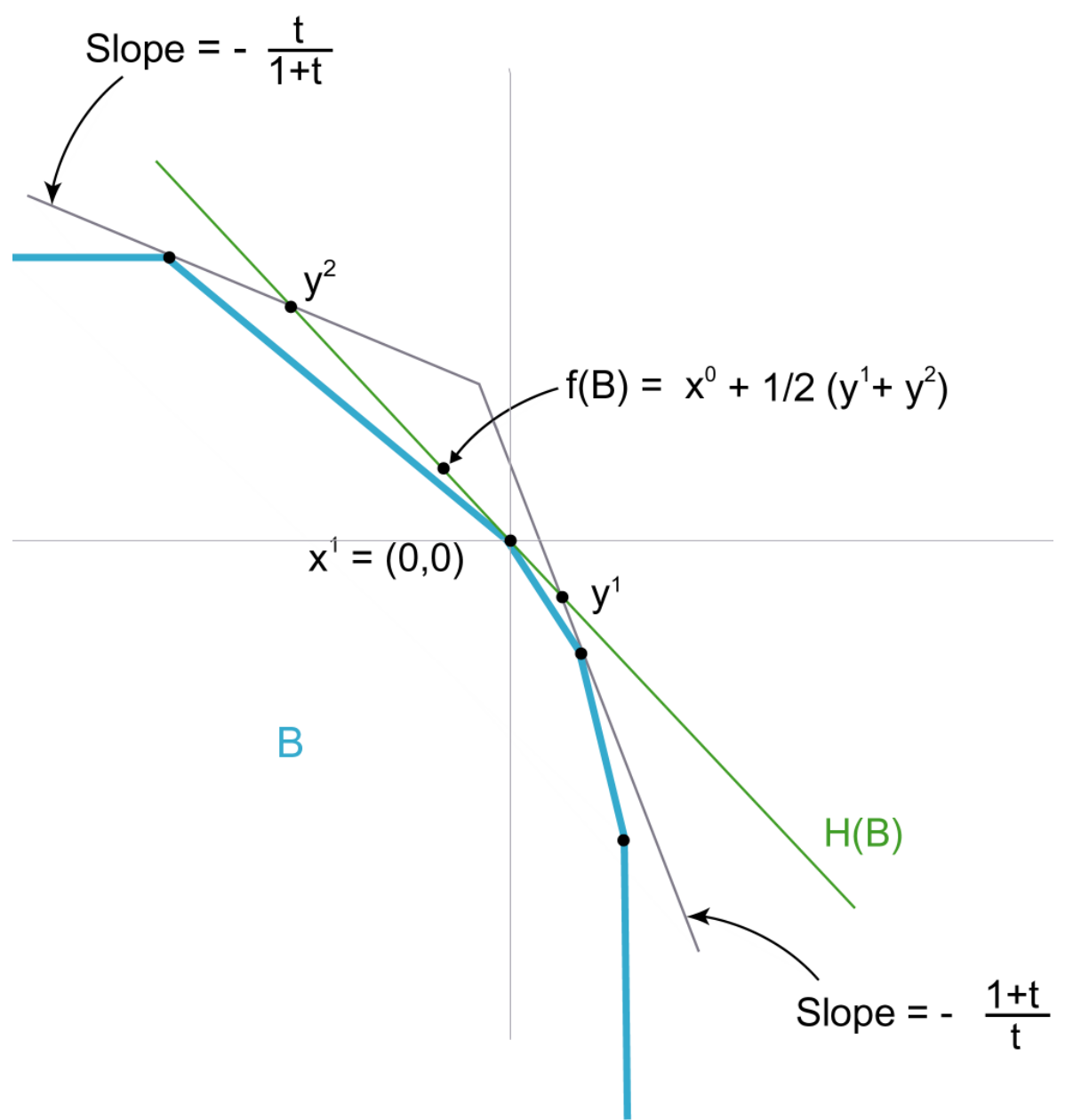

Figure 3 


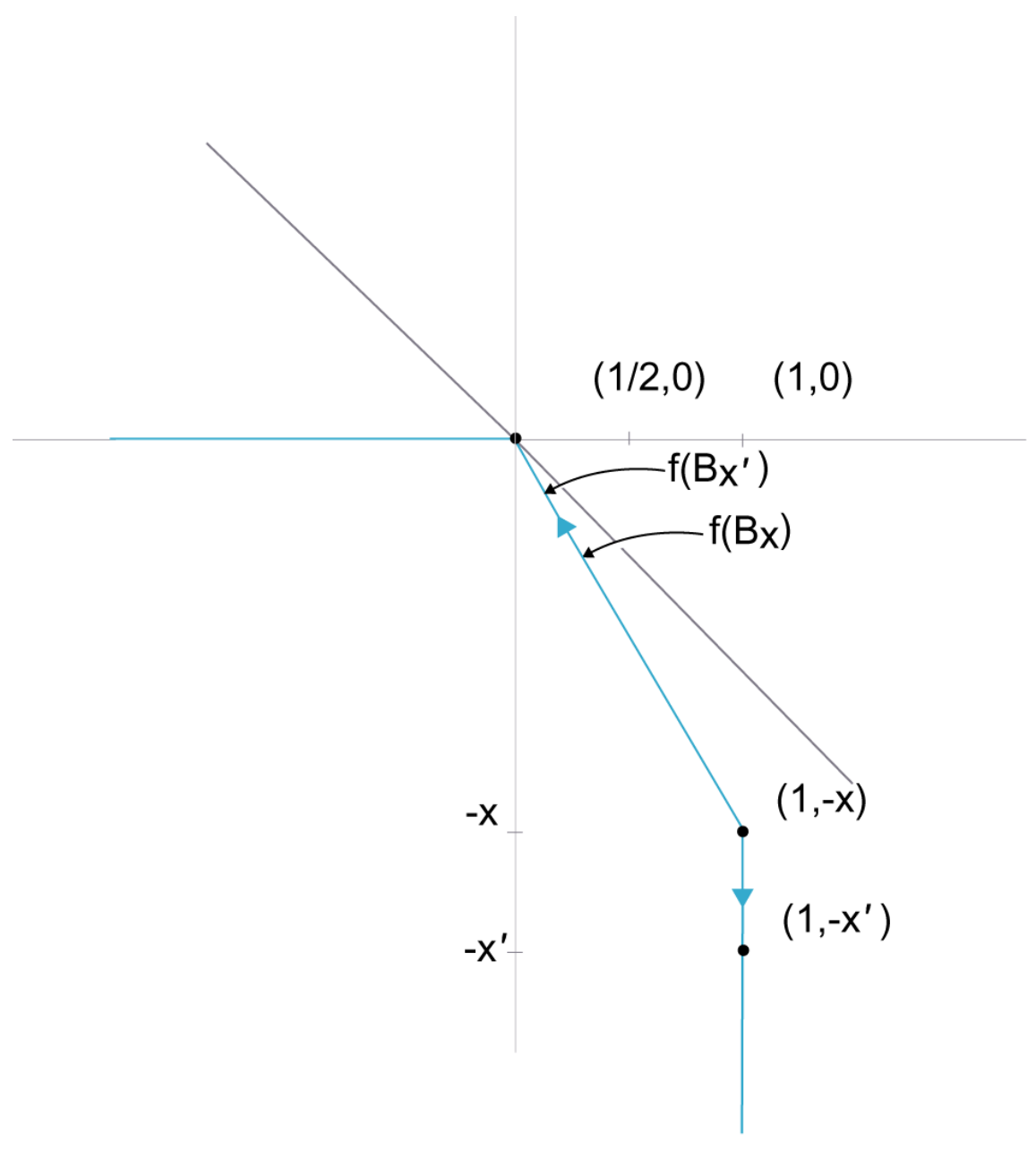

Figure 4 


\section{References}

[1] Arrow, K.J., A.K. Sen, and K. Suzumura eds., (2002), Handbook of Social Choice and Welfare

[2] Aumann, Robert J., (1989), Lectures on Game Theory, Westview, Boulder, Colorado

[3] Aumann, Robert J. and Michael Maschler, (1985), Game Theoretic Analysis of a Bankruptcy Problem from the Talmud, Journal of Economic Theory, 36, 195-213

[4] Bar-Hillel, Maya and Menachem Yaari, (1992), Judgments of Distributive Justice, chapter 4, 55-89, in Psychological Perspectives on Justice, Barbara A. Mellers and Jonathan Baran, eds., Cambridge University Press.

[5] Champsaur, Paul, (1975), How to Share the Cost of a Public Good?, International Journal of Game Theory, 4, 113-129

[6] Chugh, Dolly, Jerry Green and Lorraine Idson....

[7] Chun, Y., (1986), The Solidarity Axiom for Quasi-Linear Social Choice Problems, Social Choice and Welfare, 3, 297-320

[8] Chun, Y., (1989), Monotonicity and Independence Axioms for Quasi-linear Social Choice Problems, Seoul Journal of Economics, 2, 225-244.

[9] Chun, Y., (2000), Agreement, Separability and other axioms for Quasilinear Social Choice Problems, Social Choice and Welfare, 17, 507-521

[10] Dubins, Lester F. (1977), Group Decision Devices, American Mathematical Monthly, May, 350-356

[11] Elster, Jon, (1992), Local Justice: how institutions allocate scarce goods and necessary burdens, Russell Sage Foundation, New York

[12] Green, J. (1983), A Theory of Bargaining with Monetary Transfers, Harvard Institute of Economic Research Discussion Paper No. 966, (February).

[13] Grünbaum, Branko, (with the cooperation of Victor Klee, M. A. Perles, and G. C. Shephard), (1987), Convex Polytopes, Interscience, London.

[14] Hart, Sirgiu, (1985), An Axiomatization of Harsanyi's Non-Transferable Utility Solution," Econometrica 53, 1295-1313.

[15] Kalai, Ehud, (1977), Proportional Solutions to Bargaining Situations: Interpersonal Utility Comparisons, Econometrica, 45(7), 1623-30

[16] Kalai, Ehud and M. Smorodinsky, (1975), Other Solutions to Nash's Bargaining Problem, Econometrica, 43(3), 513-518 
[17] Kaneko, M., (1977), The Ratio Equilibrium and a Voting Game in a Public Good Economy, Journal of Economic Theory, 16(2), 123-136

[18] Loehmann, E. and Andrew Whinston, (1974), An Axiomatic Approach to Cost Allocation for Public Investment, Public Finance Quarterly, 2, 236251

[19] Maschler, Michael and Micha A. Perles, (1981), The Superadditive Solution for the Nash Bargaining Game, International Journal of Game Theory, 10(314), 163-193

[20] Maschler, Michael and Micha A. Perles, (1981), The Present Status of the Super-Additive Solution, in Essays in Game Theory and Mathematical Economics in Honor of Oskar Morgenstern, R.J. Aumann, ed., Mannheim: Bibliographisches Institut

[21] Moulin, Herve, (2001), Axiomatic Cost and Surplus-Sharing, Chapter 6 in the Handbook of Social Choice and Welfare, volume 1, K. Arrow, A.K. Sen, and K. Suzumura, eds.

[22] Moulin, Herve, (1989), Monotonic Surplus Sharing: Characterization Results, Games and Economic Behavior, 1, 250-274

[23] Moulin, Herve, (1985), The Separability Axiom and Equal-Sharing Methods, Journal of Economic Theory, 36, (1), 120-148

[24] Moulin, Herve, (1985), Egalitarianism and Utilitarianism in Quasi-Linear Bargaining, Econometrica, 53, 49-67

[25] Moulin, Herve, (1987), The Pure Compensation Problem: Egalitarianism Versus Laissez-Fairism, Quarterly Journal of Economics, 102, (4), 769-783.

[26] Moulin, Herve, (1985), Quasi-Egalitarian Cost Sharing of a Public Good, Virginia Polytechnic Institute, discussion paper E85-09-02

[27] Moulin, Herve, (1988), Axioms of Cooperative Decision Making, Cambridge University Press, Cambridge, UK

[28] Myerson, Roger B., (1981), Utilitarianism, Egalitarianism, and the Timing Effect in Social Choice Problems, Econometrica, 49 (4), 883-897

[29] Nash, John F., (1950), The Bargaining Problem, Econometrica, 18, 155-162

[30] Peters, Hans J.M., (1992), Axiomatic Bargaining Game Theory, Theory and Decision Library, Kluwer Academic Publishers, Dordrecht

[31] Peters, Hans J.M., (1986), Simultaneity of Issues and Additivity in Bargaining, Econometrica, 54, (1), 153-169

[32] Ransmeier, J.S., (1942), The Tennessee Valley Authority, Vanderbilt University Press, Nashville 
[33] Roemer, John E., (1996), Theories of Distributive Justice, Harvard University Press, Cambridge, Massachusetts

[34] Roth, Alvin E., (1979), Axiomatic Models of Bargaining, Lecture Notes in Economics and Mathematical Systems, Springer-Verlag, Berlin

[35] Roth, Alvin E., (1985), Game-Theoretic Models of Bargaining, Cambridge University Press, New York

[36] Roth, Alvin E., (1980), An Impossibility Result Concerning n-Person Bargaining Games, International Journal of Game Theory, 8, (3), 129-132

[37] Schmitz, Norbert, (undated), Two-Person Bargaining Without Threats A Review Note, Methods of Operations Research

[38] Schmeidler, David, (1969), The Nucleolus of a Characteristic Function Game, SIAM Journal of Applied Mathematics, 17, 1163-1170

[39] Sen, Amartya K., (1984), Collective Choice and Social Welfare, NorthHolland, New York

[40] Sobolev, A.I., (1975), Characterization of the Principle of Optimality for Cooperative Games Through Functional Equations, in Mathematical Methods in the Social Sciences, Vipusk 6, N.N. Vorby'ev, ed. USSR: Vilnius, 92-151 (in Russian) Preface

[41] Straffin, P.D. and J.P. Heaney, (1981), "Game Theory and the Tennessee Valley Authority", International Journal of Game Theory, 10, 35-43

[42] Tadenuma, Koichi and William Thomson , (1990), The Fair Allocation of an Indivisible Good When Monetary Compensations Are Possible, this version, University of Rochester working paper no. 257

[43] Thomson, William, (1989), Axiomatic Theory of Bargaining with a Variable Number of Players, Cambridge University Press, New York

[44] Thomson, William, (1995), Axiomatic Analysis of Bankruptcy and Taxation: A Survey, University of Rochester discussion paper no 413, October 1995

[45] Thomson, William and Roger B. Myerson, (1980), Monotonicity and Independence Axioms, International Journal of Game Theory, 9, (1), 37-49

[46] Thomson, William, (1981), Nash's Bargaining Solution and Utilitarian Choice Rules, Econometrica, 49, (2), 535-538

[47] Thomson, William, (1989), The Consistency Principle, University of Rochester, working paper no. 192.

[48] Thomson, William, (1983), Problems of Fair Division and the Egalitarian Solution, Journal of Economic Theory, 31, 211-226 
[49] Young, H. Peyton, ed., (1980), Fair Allocation, American Mathematical Society Short Course, volume 33, American Mathematical Society, Providence, Rhode Island

[50] Young, H. Peyton (1994), Equity - In Theory and Practice, Princeton University Press, Princeton, New Jersey

[51] Young, H. Peyton (1985), Monotonic Solutions of Cooperative Games, International Journal of Game Theory, 14, 65-72

[52] Young, H. Peyton (1994), "Cost Allocation", in Handbook of Game Theory with Economic Applications, Volume II, R.J. Aumann and S. Hart (eds.), North-Holland 1193-1235 